\title{
The effect of auxiliary energy on rhein, kaempferol and astragalin extraction from Cassia alata
}

\author{
Yi Ling Yeong ${ }^{1}$, Sook Fun Pang ${ }^{1,2}$ and Jolius Gimbun ${ }^{1,2 *}$ \\ ${ }^{1}$ Faculty of Chemical \& Natural Resources Engineering, Universiti Malaysia Pahang, 26300 Gambang, Pahang, Malaysia \\ ${ }^{2}$ Centre of Excellence for Advanced Research in Fluid Flow, Universiti Malaysia Pahang, 26300 Gambang, Pahang, Malaysia
}

\begin{abstract}
This paper presents the effect of auxiliary energy on rhein, kaempferol and astragalin extraction from Cassia alata. The effect of auxiliary energy was examined by performing extraction using either the ultrasonic assisted extraction (UAE) or microwave assisted extraction (MAE). A dried plant material with size ranging from $125 \mu \mathrm{m}$ to $800 \mu \mathrm{m}$ was used throughout this work. The rhein, kaempferol and astragalin quantification and identification was performed using ultra-performance liquid chromatography coupled with quadrupole time-of-flight mass spectrometer. The presence of rhein, kaempferol and astragalin were identified and compared with external standards. The residence time, neutral mass and mass fragmentation pattern between the standard and plant extract are also observed. It was found that the extraction yield of rhein, kaempferol and astragalin were higher with UAE method compared to MAE method due to degradation of active component occur. The findings in this work may serve as a useful guide to select the extraction method used to maximise the yield of rhein, kaempferol and astragalin extraction from C. alata.
\end{abstract}

\section{Introduction}

Cassia alata (Family: Leguminosae) is a beautiful flowering shrub that grows about 2 to $3 \mathrm{~m}$ in height. The leaves are pinnate and 40 to 60 centimetres long. $C$. alata produces beautiful yellow flowers in a column that look like yellow candlesticks. Due to its beauty, it is often cultivated as an ornamental plant. It is well known as ringworm shrub or ringworm Cassia in many countries due to the application of its fresh leaves in the treatment of skin diseases such as ringworm, itching, ulcers, eczema, scabies and pruritus. It is widely distributed in many tropical countries such as Malaysia, Indonesia, India, America, Fiji, Brazil and Africa. [13][4]. This herbal medicine has been recognized for centuries as a traditional medicine owing to its remarkable medical properties [2]. In Nigeria, the leaves of $C$. alata are used in the skin infection treatment and the juice expressed from the young leaves can be applied topically to the affected parts [1]. In South America, it is used for skin diseases, asthma, snake bites, fever, stomach problems and venereal disease. In Africa, the boiled leaves are used to treat hypertension, whereas in Thailand, the leaves are boiled and drunk to hasten delivery. Moreover, C. alata also used as a cure for poisonous bites and for venereal eruptions in India [4]. The leaf extract not only act as antibacterial and antifungal but also perform anti-inflammatory and antioxidant functions. The extracts of $C$. alata have been used in cosmetic product as a formulation for dermatological skin care products [3].
The major bioactive components of $C$. alata are anthraquinones and polyphenols. The leaves of $C$. alata have been qualitatively analysed for the presence of pharmacologically active anthraquinones which are rhein, aloe-emodin, chrysophanol, emodin, and physcion. These anthraquinones perform various functions which are antioxidant, antitumor, antimicrobial, antifungal, cytotoxic, and hypoglycaemic activities. Besides that, $C$. alata also contain flavonoid kaempferol which has been reported to have anticancer properties in several studies [2].

The extraction method is crucial as it will influence the yield, recovery and quality of the bioactive components in the final extract [4-7]. Previously, researchers had applied soxhlet extraction and maceration to extract bioactive components in $C$. alata [8-11]. The aforementioned technique belongs to a conventional method which needs higher temperatures and longer time in order to obtain the extract. Apart from that, higher temperature that is often used in conventional extraction process may cause a thermal degradation of the bioactive components. An alternative extraction method, Ultrasonic Assisted Extraction (UAE) was carried out by Sousa et al. [12] as this method can reduce the mass transfer limitation for both internal and external transport and with the aid of the ultrasonic wave, cell membrane of the plant may break hence facilitating release of bioactive components to the bulk liquid. However, to our knowledge, there is no study in the literature on the extraction of bioactive components from $C$. alata by using Microwave Assisted Extraction (MAE). MAE has shown to be more efficient for 
extraction of bioactive components than soxhlet, maceration and UAE because of short operating times [4]. MAE enhances the efficiency of extraction as compared to conventional extraction since microwaves interact with the polar molecules in the extraction media, heat is generated and the internal pressure of the solid material is increased [5, 13]. Yet, other bioactive components such as rhein, kaempferol and astragalin are affected by microwave irradiation or high temperature, resulting in degradation [4]. This leads to the necessity of developing this method for extraction in $C$. alata hence this is the aim of this work.

\section{Materials and methods}

\subsection{Chemicals and plant material}

Standard rhein, kaempferol and astragalin were obtained from Sigma-Aldrich whereas analysis grade ethanol was obtained from Merck (Darmstadt, Germany). HPLC grade trifluoroacetic acid (TFA) and acetonitrile was obtained from Fisher Scientific (Leicestershire, UK) and Merck (Darmstadt, Germany), respectively. C. cassia was procured from a trader in Sungai Lembing, Malaysia. The dried plant was grounded into powder and the moisture content was determined using a moisture analyzer (AND MS-70, Japan). The powdered plant was preserved in an airtight plastic at room temperature to prevent moisture absorption prior to extraction.

\subsection{Ultrasonic assisted extraction (UAE)}

Extracts were prepared using Qsonica Q700 (Newtown, USA) equipped with a standard probe. A $100 \mathrm{~mL}$ of solvent (water and 50\% ethanol) was added into weighted powder (dry weight) at the solid-to-solvent ratio of $0.05 \mathrm{~g} / 100 \mathrm{ml}$. It was understood from Pang et al. [14] that $50 \%$ aqueous ethanol solvent provides an excellent extraction of both the methoxylated and hydroxylated phenolic compound. Thus the same solvent is used in this work. The mixture was then immediately sonicated at the amplitude of $90 \%$ for the extraction time of $9 \mathrm{~min}$. The supernatant was then separated by centrifugation (Eppendorf 5810 R, Hamburg, Germany) at $10000 \mathrm{rpm}$ for $15 \mathrm{~min}$ to obtain a clear solution. The extracts were then stored at $-80^{\circ} \mathrm{C}$ freezer to prevent the degradation of bioactive components in the extract.

\subsection{Microwave assisted extraction (MAE)}

Extracts were prepared using SP-Microwave (CEM) which is controlled by Synergy ${ }^{\mathrm{TM}}$ software using a method adapted from our earlier work [14]. A $5 \mathrm{~mL}$ of solvent (water and 50\% ethanol) was added into weighted powder (dry weight) at the solid-to-solvent ratio of $0.05 \mathrm{~g} / \mathrm{ml}$. The mixture was then immediately put in the microwave at the power of $200 \mathrm{~W}$ and extracted for 5 min. The supernatant was then separated by centrifugation (Eppendorf 5810 R, Hamburg, Germany) at $10000 \mathrm{rpm}$ for $15 \mathrm{~min}$ to obtain a clear solution. The extracts were then stored at $-80^{\circ} \mathrm{C}$ freezer to prevent the degradation of bioactive components in the extract.

\subsection{Quantification of rhein, kaempferol and astragalin}

The quantification of kaempferol, rhein, and astragalin was performed on Waters UPLC-TUV-QTOF Xevo G2S. Waters Acquity UPLC I-Class (Waters Corporation, Milford, USA) fitted with Acquity UPLC HSS T3 column $(2.1 \times 75 \mathrm{~mm}, 1.8 \mu \mathrm{m})$ and a Acquity UPLC HSS T3 VanGuard column guard $(2.1 \times 5 \mathrm{~mm}$, $1.8 \mu \mathrm{m})$ was used for analysis. The column temperature is $30^{\circ} \mathrm{C}$ with injection volume of $1 \mu \mathrm{L}$ and flowrate at 600 $\mu \mathrm{L} / \mathrm{min}$. The mobile phase consists of water in $0.1 \%$ formic acid (solvent $\mathrm{A}$ ) and acetonitrile in $0.1 \%$ formic acid (solvent B). The performed elution scheme were: 0$2.0 \mathrm{~min}, 85 \%$ A; 2-6 min, 50\% A; 6-10 min, 5\% A; $10-$ $11 \mathrm{~min}, 85 \% \mathrm{~A}$; and the MS system was performed on Waters Xevo G2S QTOF with ionization ESI negative mode, capillary voltage of $1.5 \mathrm{kV}$. The collision energy is ramp from 10 to $40 \mathrm{eV}$ and acquisition mass range from 100 to $1500 \mathrm{~m} / \mathrm{z}$.

\section{Results and discussion}

\subsection{Comparison of ultraviolet and mass spectrometry of MAE extract}

The extracts obtained in $C$. alata by using UAE and MAE were analysed by using UPLC-TUV-QTOF MS. This is an analytical technique in which the separation performed by liquid chromatography and analyses with UV detector and a mass spectrometer. This technique is high in selectivity and sensitivity, hence often used for plant sample characterization. Fig. 1. Show the UV chromatogram and MS chromatogram of MAE extract. More compound peaks are detectable under MS compared to UV. UPLC-QTOF MS has an advantage over the other method such as GC-MS because it can be used to separate the plant extracts containing compounds that are highly polar and less volatile [9]. Thus, separation of phenolic compounds in the $C$. alata extracts was conducted via LC-MS and LC-UV. It is known that the sensitivity of LC-MS is 10 times more than that of LC-UV. Therefore, LC-MS is preponderance for microanalysis. Apart from that, LC-MS can identify the component by retention time $(\mathrm{t}(\mathrm{R}))$ and $\mathrm{m} / \mathrm{z}$, whereby it has high selectivity and exclusion for the determination of bioactive component. Meanwhile, the LC-UV is simpler, lower cost and has a better separation of components. Therefore, it is preferred for identification of abundant component, which is easier to separate [15]. Owing to the nature of the sample in this work, the UPLC-QTOF-MS was used to compare the active compounds obtained from MAE and UAE. 


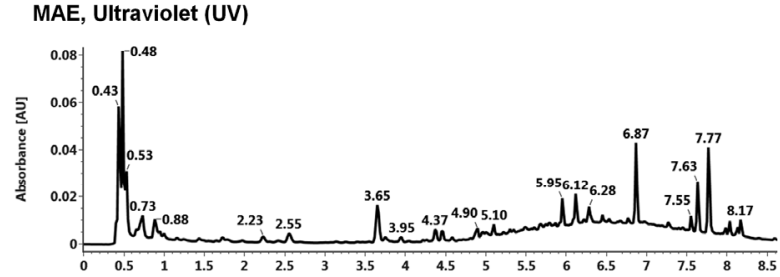

MAE, Mass Spectrometry (MS)

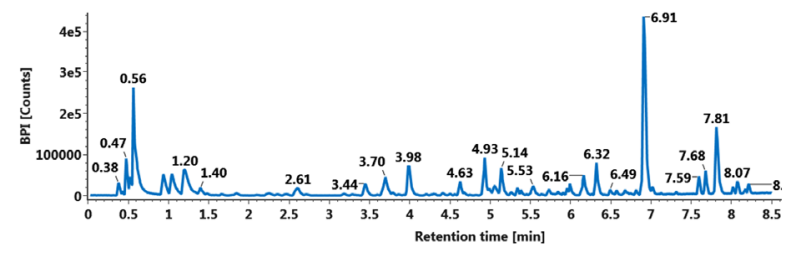

Fig. 1. Microwave assisted extraction (MAE) Comparison of Ultraviolet (UV) and Mass Spectrometry (MS).

\subsection{Mass spectrometry comparison between the MAE and UAE extract}

The sonication in UAE produced the mechanical waves that will generate micro-cavitation in the liquid surrounding the plant material, disrupting the plant cell wall and releasing the extract. The kinetic energy is introduced to the whole bulk liquid volume by the cavitation bubbles collapse at the interface, hence increasing the mass transfer across the sold-liquid interface. This will induce penetration of the solvent into cell membrane walls and facilitating the release of cells contents and improving the mass transfer [16].

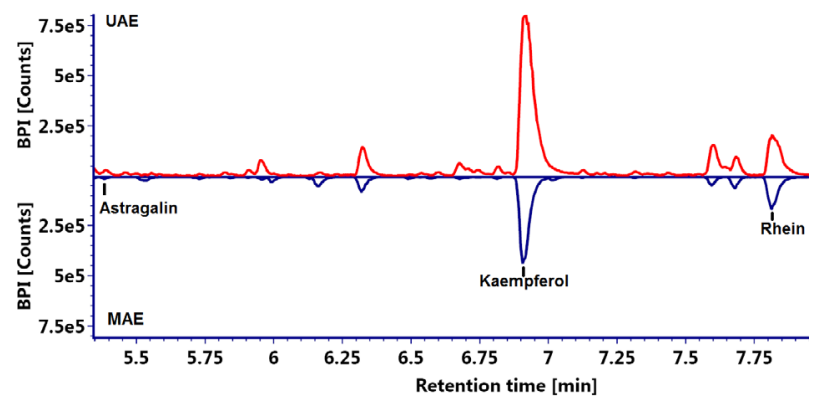

Fig. 2. Comparison of UAE and MAE in liquid chromatography mass spectrometry (LCMS) base peak ion chromatogram. UAE (red line), MAE (blue line).

The base peak ion chromatogram of MAE and UAE extract were compared in Fig. 2. It can be observed that the chemical profile of the MAE extracts is slightly different from that of UAE extracts. The three major compounds, namely, kaempferol, rhein and astragalin were identified by comparing the retention times, UV spectra and MS data of the separated compounds with their respective standards. Kaempferol elutes at 6.92 minutes, rhein elutes at 7.82 minutes and astragalin elutes at 5.39 minutes. The MAE is an efficient method for extraction of bioactive components from plant materials because of its shorter operational times. However, rhein, kaempferol and astragalin may be affected by the microwave irradiation or high temperature, resulting in degradation which adversely affecting the yield [4]. Therefore, the yield of extraction by MAE is not always higher than that of UAE.

\subsection{Comparison of rhein, kaempferol and astragalin yield}

The yield of bioactive components such as kaempferol, rhein, and astragalin by using UAE is higher than that of MAE as shown in Fig. 3. The yield of kaempferol and astragalin by UAE is doubled to that of MAE. For instance, UAE yielded $0.32 \mathrm{mg} / \mathrm{g}$ of kaempferol whereas only $0.14 \mathrm{mg} / \mathrm{g}$ was obtained from MAE. Similarly for astragalin the yield from UAE $(0.02 \mathrm{mg} / \mathrm{g})$ is doubled to that of MAE $(0.01 \mathrm{mg} / \mathrm{g})$. The different between UAE $(0.09 \mathrm{mg} / \mathrm{g})$ and MAE $(0.06 \mathrm{mg} / \mathrm{g})$ on the yield of rhein is not a much as that of kaempferol and astragalin, although it is clear that UAE is a better method for extracting rhein than MAE. As mentioned earlier this is due to cell disruption by sonication in UAE which enhanced the release of active compound from the plant material.
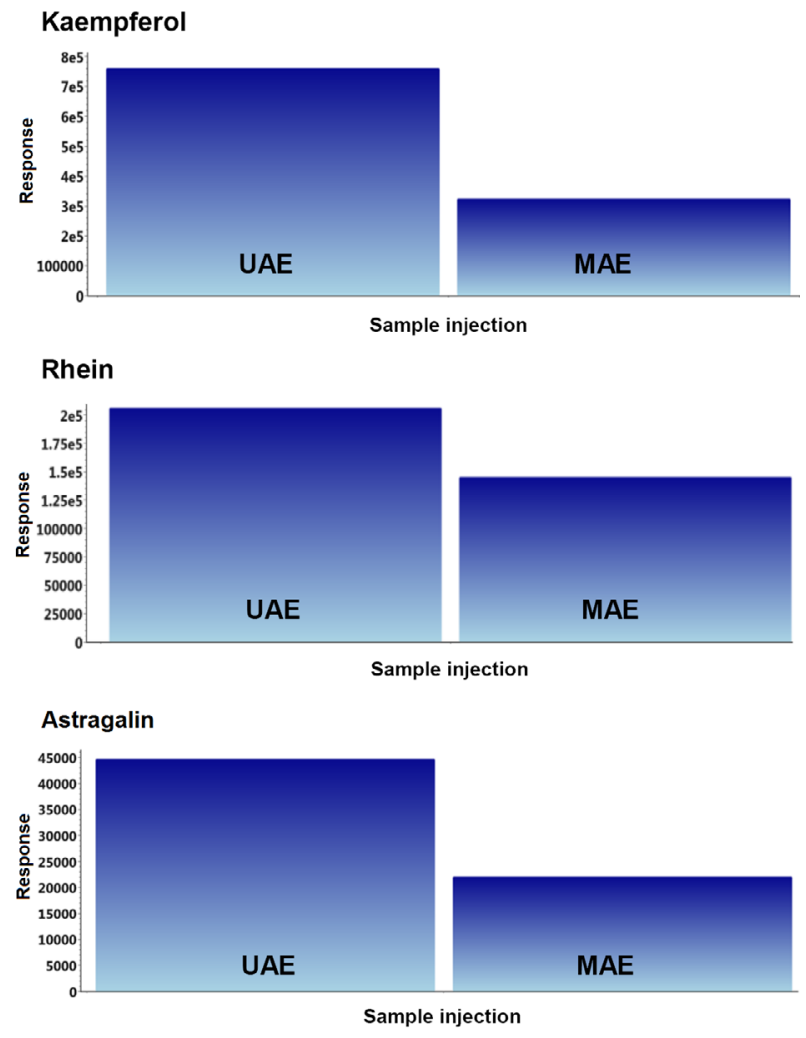

Fig. 3. Comparison of the yield of bioactive components by using UAE and MAE extraction method.

In the microwave extraction, the atomic kinetic energy is introduced and the heat propagates in the liquid phase, hence increasing the diffusion rate, while sonication introduced the kinetic energy to the whole volume as the cavitation bubbles collapse at the interface hence improving the mass transfer across the solid-liquid interface. Compared to MAE, UAE has the main advantage of working closer to ambient temperatures, thus avoiding the thermal overexposure [17]. In this case, rhein kaempferol and astragalin may suffer from 
thermal degradation induced by the excessive heat from the microwave irradiation in MAE.

\section{Conclusion}

The liquid chromatography with mass spectrometry detector method developed in this work for $C$. alata extracts had successfully identified and confirmed the presence of rhein, kaempferol and astragalin in the extracts by matching the mass spectra and residence time obtained from the plant extracts and external standard. It was found that UAE provide a higher extraction yield of rhein, kaempferol and astragalin from $C$. alata leaves compared to MAE. In this work the sonication increased the yield of bioactive component extraction from $C$. alata by at least $30 \%$ compared to that of MAE.

\section{References}

1. P. L. Rajagopal, R. Majetha, K .R. Sreejith, S. S. Kiron, S. Aneeshia, K. Premaletha, J. Int. Aca. Res. Multidiscip. 2, 280-307 (2014)

2. V. E. Fernand, D. T. Dinh, S. J. Washington, S. O. Fakayode, J. N. Losso, R. O. V. Ravenswaay, I. M. Warner, Talanta. 74, 896-902 (2008)

3. D. E. Okwu and F. U., Nnamdi, Pelaga. Res. Lib. 2(2), 247-254 (2011)

4. C. A. L. Escobar, F. P. Capote, M. D. L. de Castro, Talanta. 144, 522-528 (2015)

5. S. F. Pang, M. Z. Lau, M. M. Yusoff, J. Gimbun, Mater. Sci. Forum (890), 155-158 (2017)

6. J Sandanasamy, M. M. Yusoff, J. Gimbun, Mater. Sci. Forum (890), 167-170 (2017)

7. S. L. Nguang, Y. L. Yeong, S. F. Pang, J. Gimbun, Ind. J. Sci. Technol. (10)2, (2017)

8. W. F. Sule, I. O. Okonko, S. Omo-Ogun, J. C. Nwamze, M. O. Ojezele, O. J. Ojezele, J. A. Alli, E. T. Soyemi, T. O. Olaonipekun, J. Med. Plants Res. 5(2), 176-183 (2011)

9. W. Gritsanapan and P. Mangmeesri, J. Health. Res. 23(2), 59-64 (2009)

10. C. A. Alalor., C. I. Igwilo., E. Jeroh, Asian. J. Biol. Sci. (5), 120-125 (2012)

11. O. Oladeji., F. Adelowo., K. Odelade., S. Aremu., M. Adisa, CIBTech. J. Pharma. Sci. 5(3), 40-51 (2016)

12. A. D. Sousa, A. I. V. Maia, T. H. S. Rodrigues, K.M. Canuto, P. R. V. Ribeiro, R. D. C. A. Pereira, R. F. Vieira, E. S. de Brito, Ind. Crops Prod. (79), 91-103 (2016)

13. A. E. Ince, S. Sahin, G. Sumnu, J. Food. Sci. Technol. 51(10), 2776-2782

14. S. F. Pang, M. M. Yusoff, J. Gimbun, Food Hydrocoll. (37), 159-165, (2014)

15. R. H. Liu, A L. Jiang, L. L. Bian, F. Shao, Z. L. Ma, B. Y. Yu, US Nat. Lib. Med. 30(3), 285-289 (2007)
16. A. Alupului, I. Calinescu, V. Lavric, AIDIC. Conference. Series. (9), 1-7 (2009)

17. V. Mandal, Y. Mohan, S. Hemalatha, Pharma Rev. 1, 7-18 (2007) 